\title{
BMJ Open Prognostic value of 12 novel cardiological biomarkers in stable coronary artery disease. A 10-year follow-up of the placebo group of the Copenhagen CLARICOR trial
}

To cite: Winkel P, Jakobsen JC, Hilden J, et al. Prognostic value of 12 novel cardiological biomarkers in stable coronary artery disease. A 10-year follow-up of the placebo group of the Copenhagen CLARICOR trial. BMJ Open 2020;10:e033720. doi:10.1136/ bmjopen-2019-033720

- Prepublication history and additional material for this paper are available online. To view these files, please visit the journal online (http://dx.doi org/10.1136/bmjopen-2019033720).

Received 28 August 2019 Revised 22 April 2020 Accepted 17 June 2020

A) Check for updates

(c) Author(s) (or their employer(s)) 2020. Re-use permitted under CC BY-NC. No commercial re-use. See rights and permissions. Published by BMJ.

For numbered affiliations see end of article.

Correspondence to

Dr Per Winkel; pwinkel@ctu.dk

\section{ABSTRACT}

Objective To assess if 12 novel circulating biomarkers, when added to 'standard predictors' available in general practice, could improve the 10-year prediction of cardiovascular events and mortality in patients with stable coronary heart disease.

Design The patients participated as placebo receiving patients in the randomised clarithromycin for patients with stable coronary artery disease (CLARICOR) trial at a random time in their disease trajectory.

Setting Five Copenhagen University cardiology departments and a coordinating centre.

Participants 1998 participants with stable coronary artery disease.

Outcomes Death and composite of myocardial infarction, unstable angina pectoris, cerebrovascular disease and death.

Results When only 'standard predictors' were included, $83.4 \%$ of all-cause death predictions and $68.4 \%$ of composite outcome predictions were correct. Log(calprotectin) and log(cathepsin-S) were not associated $(p \geq 0.01)$ with the outcomes, not even as single predictors. Adding the remaining 10 biomarkers (high-sensitive assay cardiac troponin $\mathrm{T}$; neutrophil gelatinase-associated lipocalin; osteoprotegerin; N-terminal pro-B-type natriuretic peptide; tumour necrosis factor receptor 1 and 2; pregnancy-associated plasma protein A; endostatin; YKL40; cathepsin-B), which were all individually significantly associated with the prediction of the two outcomes, increased the figures to $84.7 \%$ and $69.7 \%$. Conclusion When 'standard predictors' routinely available in general practices are used for risk assessment in consecutively sampled patients with stable coronary artery disease, the addition of 10 novel biomarkers to the prediction model improved the correct prediction of allcause death and the composite outcome by $<1.5 \%$. Trial registration number NCT00121550.

\section{Strengths and limitations of this study}

Use of multiple biomarkers.

- Well-established cohort.

- Comprehensive statistical approach.

- Missing external validation.

Relatively old cohort.

\section{INTRODUCTION}

Previously, we have studied the prognostic impact of routinely available 'standard predictors' when added to a prediction model void of covariates using the placebo receiving participants from the CLARICOR trial. ${ }^{1-4}$ The impact, however, was quite modest. ${ }^{1}$ For risk assessment of patients with coronary artery disease (CAD), there are a number of advanced biomarkers, including several from outside cardiology, which may help identifying CAD patients at high risk of cardiovascular (CV) disease manifestations. ${ }^{2}$ Here, we assess the prognostic impact-relative to standard clinical predictors usually available during routine clinical work-of 12 newer biomarkers in predicting death and other serious CV events in patients suffering from CAD sampled while their disease was stable.

Briefly, the biomarkers are (1) serum N-terminal pro-B-type natriuretic peptide (pro$\mathrm{BNP}$ ), a marker of left ventricular dysfunction and heart failure; (2) high-sensitive assay cardiac troponin $\mathrm{T}$ (hs-cTnT) indicating myocardial ischaemia; (3) YKL40 found to be predictive of acute myocardial infarction (AMI), CV death and non-CV death; (4) the glycoprotein osteoprotegerin (OPG), which is positively related to coronary calcification, 
vascular stiffness and the presence of unstable atherosclerotic plaques; (5) pregnancy-associated plasma protein A (PAPP-A), a marker of vulnerable plaques in coronary arteries; (6) cathepsin-B and (7) cathepsin-S, a group of proteinases that have been suggested to be causally involved in the different stages of the atherosclerotic process; (8) endostatin, an endogenous angiogenesis inhibitor suggested to mirror an increased neovascularisation induced by vascular or myocardial ischaemia; the soluble receptors, (9) soluble tumour necrosis factor receptor 1 (sTNFR1) and (10) soluble TNF receptor 2 (sTNFR2), suggested to portray information about a systemic inflammatory state that is independent of other more established inflammatory markers; (11) calprotectin and (12) neutrophil gelatinase-associated lipocalin (NGAL), both released from neutrophils when the cells are activated. Circulating levels of neutrophils and their activation products have been shown to be markers for plaque instability in both primary and secondary prevention of CV diseases.

All of these have been claimed to add some prognostic information in patients with stable coronary artery disease. Our group has tested the individual importance of many of these biomarkers, and in many studies statistical inference supports the view that biomarkers may improve the prediction. ${ }^{5-12}$

Our objectives were to clarify: (1) which of these newer biomarkers maintain their prognostic importance if all of them were simultaneously available and were combined with the routinely available clinical and laboratory information, and (2) what would then be their relative practical contribution if they were added to the 'standard predictors' such as age, smoking, plasma lipids, etc. In accordance with our published statistical analysis plan, ${ }^{2}$ our analysis focusses on all-cause death and on a composite outcome comprising AMI, unstable angina pectoris (UAP), cerebrovascular disease (CeVD) and death.

\section{MATERIALS}

\section{The patients}

The study population is the placebo patients from the CLARICOR study. ${ }^{3}{ }^{4}$ Patients aged 18-85 years, from the Copenhagen area, who had a discharge diagnosis of myocardial infarction or angina pectoris during 19931999 and were alive in August 1999 were invited by letter for an interview and a 14-day trial of clarithromycin versus placebo. ${ }^{34}$ Out of the 4372 who were randomised during October 1999 through April 2000, 2200 were in the placebo group.

The main results of the trial were that clarithromycin increased the risk of $\mathrm{CV}$ as well as all-cause death. ${ }^{13-15}$ Therefore, we here focus on the placebo group.

For the CLARICOR trial, only patients who were in a stable state of their coronary heart disease were selected. Thus, patients were excluded if they fulfilled one or more of the following conditions: (1) had suffered from AMI or UAP within the previous 3 months; (2) had intracoronary interventions within the previous 6 months; (3) had impaired renal function; (4) had hepatic dysfunction; (5) had congestive heart failure (New York Heart Association IV classification of heart failure); (6) had active malignancy; (7) were without capacity to manage own affairs; (8) were breast feeding and (9) were possibly pregnant.

Of the 2200 participants one had garbled study data, and further 201 had one or more missing biomarker measurements (see below), leaving 1998 participants for the present analysis. Only 15 of these were lost track of due to emigration or disappearance.

\section{The predictors}

Information on smoking status, current medication, known hypertension, diabetes, sex, age and myocardial infarction at index hospitalisation or UAP was obtained from the local hospital files and patient interviews.

\section{Biochemical measurements on serum collected at enrolment visit}

Biochemical data were obtained from analysis of serum specimens sampled at inclusion of the patients and stored at $-80^{\circ} \mathrm{C}$. The quantities measured include lipoproteins, ${ }^{16}$ high-sensitivity-C-reactive-protein $/ \mathrm{mg} / \mathrm{L}^{7}$ and glomerular filtration rate $/ \mathrm{mL} / \mathrm{min}$ using creatinine. ${ }^{17}$ These quantities together with the variables mentioned under predictors above are those collectively referred to as 'standard predictors'.

Biomarkers included as newer biomarkers were YKL40/ $\mu \mathrm{g} / \mathrm{L}^{8}$; hs-cTnT $/ \mathrm{ng} / \mathrm{L}^{9} ;$ binary pregnancy associated plasma protein-A (binary-PAPP-A), which is coded as 1 if PAPP-A was $\geq 4 \mathrm{mIU} / \mathrm{L}$ or 0 otherwise ${ }^{10}$; pro-BNP $/ \mathrm{ng}$ / $\mathrm{L}^{9}$; cathepsin- $\mathrm{B} / \mu \mathrm{g} / \mathrm{L}^{6}{ }^{18}$; endostatin $/ \mathrm{ng} / \mathrm{mL}^{19}$; cathep$\sin -\mathrm{S} / \mu \mathrm{g} / \mathrm{L}^{620} ; \mathrm{sTNFR} 1 / \mathrm{pg} / \mathrm{mL}$ and sTNFR2/pg/mL ${ }^{521}$; $\mathrm{NGAL} / \mathrm{ng} / \mathrm{L}^{22}$; calprotectin $/ \mathrm{mg} / \mathrm{L}^{11}$ and $\mathrm{OPG} / \mathrm{ng} / \mathrm{L} .^{12}$ Due to storage problems some marker data are missing on some patients.

\section{The outcomes}

Initial follow-up of the patients lasted for approximately 2.6 years, during which outcomes were collected through hospital and death registries and assessed by an adjudication committee. ${ }^{4}$ Corresponding register data later produced similar results. ${ }^{23}{ }^{24}$ The adjudicated outcomes were therefore replaced and augmented by register outcomes to cover up to 10 years \pm 3 months of follow-up. Last register follow-up was 31 December 2009. The public registers have an almost $100 \%$ coverage and the quality of these is described elsewhere. ${ }^{25} 26$ The algorithm used to get from the International Statistical Classification of Diseases used in the national registries to the events of the composite outcome is described in detail previously. ${ }^{13}$

We assessed (1) the time from randomisation to allcause death and (2) the time from randomisation until the first occurrence of one of the following outcomes: AMI, UAP, CeVD or all-cause death. 


\section{METHODS}

\section{Statistical analysis}

The statistical principles and techniques used have previously been published. ${ }^{12}$ While our previous publication ${ }^{1}$ dealt with the prognostic impact of the 'standard predictors', we here use the same techniques to quantify the effect of adding biomarker information to the 'standard predictors'.

We used Cox regressions (SAS V.9.4), where all analyses that included covariates were stratified by centre. The assumption of proportional hazards over time covering all covariates included in a Cox analysis and the chosen functional form of quantitative covariates was tested using cumulative sums of martingale-based residuals over follow-up time and/or covariate values. ${ }^{27}$

We also analysed data using a parametric, accelerated failure-time model using the generalised gamma model of error. ${ }^{28} \mathrm{~A}$ significance level of 0.01 was used to pinpoint empirical trends worthy of note. The logarithms of the present text are natural logarithms, so whenever the predictor is a $\log$ (serum concentration/unit), the HR is the factor by which the hazard increases when the logarithm increases by 1 , that is, when the concentration increases by a factor $e=2.72$.

Biomarkers with an HR with $p$ value $\geq 0.01$ when used alone as covariate as well as when used in combination with the 'standard predictors' were excluded from further analyses. The remaining biomarkers were considered prognostic.

Assessment of the practical impact of using the set of newer biomarkers was obtained by comparing the per cent correct predictions obtained when the standard predictors were used alone with the percentage obtained when they were combined with the novel biomarkers using the method described earlier. ${ }^{1}$

Second, we report the areas under the receiver operating characteristics (ROCs), also known as area under the curves (AUCs) or C-indices, which one obtains when the Cox-Breslow risk estimates are matched against the events seen in the time window 0 -to-9 years. The muchused binary (event vs no event) $C$-index is the concordance rate between risks and outcomes. It shows how frequently an event participant has a poorer prediction score than a non-event participant. In order to reward correct prediction of time of event, we further report Harrell's 'dynamic' (or 'overall') G-index. ${ }^{29}{ }^{30}$ It shows how frequently an earlier-event participant has a poorer prediction than a later-or-never-event participant. In other words, it is the concordance between risk score and event time. It is calculated across all pairs of participants where the time order of the pair is deducible from the 9-year data window.

It is noted that in the ROC analysis it was not possible to add two time-dependent covariates, which were needed to compensate for the fact that both age and $\log (\mathrm{OPG} /$ $\mathrm{ng} / \mathrm{L}$ ) violated the assumption of proportional hazard. However, the output obtainable from the SAS procedure did not allow the inclusion of time-dependent covariates.

\section{Patient and public involvement}

There was no direct patient involvement in the design of the trial, but the majority of the investigators had daily contact with patients comparable to those included in the trial and therefore knew their needs and preferences well. Moreover, there were patient representatives as part of the regional ethics committee approving the trial.

\section{RESULTS}

Table 1 presents an overview of the covariates expected to be available from patients with stable CV disease during clinical routine work ('standard predictors') plus the 12 newer biochemical quantities under investigation. The data revealed that at 3 years, $2073(94.2 \%)$ were still alive and $1826(83.0 \%)$ had not yet suffered a composite outcome. At 6 years, 1758 (79.9\%) were still alive and 1261 $(57.3 \%)$ had not yet suffered a composite outcome. At 9 years, the numbers were $1487(67.6 \%)$ and $969(44.0 \%)$.

Out of 2099 placebo patients, 1998 had complete biochemical data. As Little's test ${ }^{31}$ had $\mathrm{p}=0.49$, suggesting that the values were missing completely at random, we used complete case analyses in the following.

Two of the 12 newer biomarkers ( $\log$ (Calprotectin) and $\log ($ Cathepsin-S) $)$ did not contribute significantly ( $>0.01)$ to the prediction of any of the two outcomes, neither when used in combination with the 'standard predictors' nor when used alone (see online supplementary tables $1 \mathrm{~S}$ and $2 \mathrm{~S}$ ). They were therefore removed from the subsequent analyses. In the analysis of $\log (\mathrm{OPG} /$ $\mathrm{ng} / \mathrm{L}$ ), we found that the assumption of proportional hazard was significantly violated. This was remedied when we included the time-dependent covariate $\log$ $(\mathrm{OPG} / \mathrm{ng} / \mathrm{L}) *$ time/year in the subsequent regression equation (see online supplementary table $2 \mathrm{~S}$ ). The latter equation now included the 'standard predictors', plus the remaining 10 newer biomarkers and the abovementioned time-dependent covariates. It appears from online supplementary table $2 \mathrm{~S}$ that only $\log$ (pro-BNP/ ng/L), $\log (\mathrm{hs}-\mathrm{cTnT} / \mathrm{ng} / \mathrm{L})$ and $\log (\mathrm{OPG} / \mathrm{ng} / \mathrm{L}) *$ time/ year contributed significantly to the prediction.

Table 2 (see also online supplementary tables $3 \mathrm{~S}$ and $4 \mathrm{~S}$ ) compares the number and percentages of correct predictions between various prediction models. In each model, predictions were made at 3, 6 and 9 years for each of the two outcomes (death and the composite). Model 1 shows the results obtained using a model void of covariates; $79.8 \%$ of the predictions were correct for the outcome death and $63.2 \%$ for the composite outcome. Model 2 shows the results obtained when model 1 was augmented by the 'standard predictors'. Now the per cent correct predictions have been improved by $83.3 \%-79.8 \%=3.5 \%$ for the outcome death and $68.4 \%-63.2 \%=5.2 \%$ for the composite outcome. When model 2 was improved by adding the 10 newer biomarkers, the additional gain in correct predictions amounted to $1.4 \%$ for death and $1.3 \%$ for the composite outcome. 
Table 1 Distributions of demographics, previous history, current medication, standard biochemical predictors and newer biochemical predictors in 2199 placebo receiving patients from the CLARICOR trial

\begin{tabular}{|c|c|}
\hline Quantity & Distribution \\
\hline \multicolumn{2}{|l|}{ Demographics and previous history } \\
\hline Sex (male) N (\%) & $1518(69.0 \%)$ \\
\hline Age/year mean (SD) & $65.2(10.4)$ \\
\hline \multicolumn{2}{|l|}{ Smoking status N (\%) } \\
\hline Smokers & $753(34.2 \%)$ \\
\hline Ex-smokers & $1011(46.0 \%)$ \\
\hline Never smoked & $435(19.8 \%)$ \\
\hline Hypertension N (\%) & $883(40.2 \%)$ \\
\hline Diabetes N (\%) & $337(15.3 \%)$ \\
\hline Previous AMI N (\%) & $1494(67.9 \%)$ \\
\hline \multicolumn{2}{|l|}{ Current medication } \\
\hline Aspirin N (\%) & $1937(88.1 \%)$ \\
\hline Beta-blocker N (\%) & $681(31.0 \%)$ \\
\hline Calcium-antagonist N (\%) & $772(35.1 \%)$ \\
\hline ACE-inhibitor N (\%) & $577(26.3 \%)$ \\
\hline Long-lasting nitrate N (\%) & $457(20.8 \%)$ \\
\hline Diuretics N (\%) & $773(35.2 \%)$ \\
\hline Digoxin N (\%) & $126(5.7 \%)$ \\
\hline Statins N (\%) & $904(41.1 \%)$ \\
\hline Anti-arrhythmic drugs $\mathrm{N}(\%)$ & $51(2.3 \%)$ \\
\hline \multicolumn{2}{|l|}{ Standard biochemical predictors } \\
\hline $\log (\mathrm{CRP} / \mathrm{mg} / \mathrm{L})$ mean $(\mathrm{SD}) \mathrm{N}^{*}$ & $1.03(1.12) 2159$ \\
\hline ApoA1/mg/dL mean (SD) $\mathrm{N}$ & $1.70(0.34) 2076$ \\
\hline $\log (\mathrm{ApoB} / \mathrm{mg} / \mathrm{dL})$ mean (SD) N & $0.16(0.27) 2075$ \\
\hline Chol-HDL/mmol/L mean (SD) $\mathrm{N}$ & $1.02(0.32) 2074$ \\
\hline Chol-LDL/mmol/L mean (SD) N & $2.56(0.72) 2079$ \\
\hline $\log ($ Cholesterol $/ \mathrm{mmol} / \mathrm{L})$ mean (SD) $\mathrm{N}$ & $1.73(0.20) 2075$ \\
\hline $\log ($ Triglyceride/mmol/L) mean (SD) N & $0.73(0.53) 2078$ \\
\hline $\begin{array}{l}\text { Glomerular filtration rate (GFR/mL/min) } \\
\text { mean (SD) N }\end{array}$ & $71.8(19.2) 2079$ \\
\hline \multicolumn{2}{|l|}{ Newer biochemical predictors } \\
\hline $\log ($ pro-BNP/ng/L) mean (SD) N & $5.26(1.37) 2149$ \\
\hline $\log (\mathrm{hs}-\mathrm{cTnT} / \mathrm{ng} / \mathrm{L})$ mean (SD) N & $2.01(0.78) 2111$ \\
\hline $\log ($ Endostatin $/ \mathrm{ng} / \mathrm{mL})$ mean $(\mathrm{SD}) \mathrm{N}$ & $10.3(0.34) 2121$ \\
\hline $\log (\mathrm{OPG}) / \mathrm{ng} / \mathrm{L})$ mean $(\mathrm{SD}) \mathrm{N}$ & $7.49(0.40) 2108$ \\
\hline $\log (T N F R 1 / p g / m L)$ mean (SD) N & $7.40(0.40) 2120$ \\
\hline $\log (\mathrm{TNFR} 2 / \mathrm{pg} / \mathrm{mL})$ mean $(\mathrm{SD}) \mathrm{N}$ & $8.54(0.33) 2120$ \\
\hline PAPP-A $\geq 4$ mIU/L count (\%) N & $288(13.1 \%) 2140$ \\
\hline $\log (Y K L 40 / \mu g / L)$ mean (SD) N & $4.75(0.66) 2163$ \\
\hline $\log (\mathrm{NGAL} / \mathrm{ng} / \mathrm{L})$ mean $(\mathrm{SD}) \mathrm{N}$ & $11.6(0.46) 2121$ \\
\hline 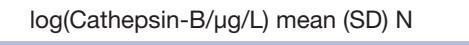 & $10.6(0.45) 2120$ \\
\hline 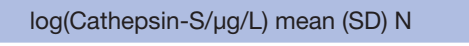 & $9.48(0.27) 2121$ \\
\hline $\log ($ Calprotectin/mg/L) mean (SD) N & $0.77(0.59) 2086$ \\
\hline
\end{tabular}

*The value of $\mathrm{N}$ varies because the laboratory tests have missing values (mostly due to storage problems). log: natural logarithm.

AMI, acute myocardial infarction; CRP, C reactive protein; $\mathrm{HDL}$, high-density lipoprotein; hs-cTnT, high-sensitive assay cardiac troponin T; LDL, low-density lipoprotein; NGAL, neutrophil gelatinase-associated lipocalin; OPG, osteoprotegerin; PAPP-A, pregnancy-associated plasma protein $\mathrm{A}$; pro-BNP, serum $\mathrm{N}$-terminal pro-Btype natriuretic peptide; TNFR1, tumour necrosis factor receptor 1; TNFR2, tumour necrosis factor receptor 1
Using the parametric model in place of the Cox model, we obtained quite similar results (see online supplementary tables $3 \mathrm{~S}$ and $4 \mathrm{~S}$ and online supplementary figure 1S A-B). The same was true if we only included $\log (-$ pro-BNP/ng/L), $\log (\mathrm{hs}-\mathrm{cTnT} / \mathrm{ng} / \mathrm{L})$ and $\log (\mathrm{OPG} /$ $\mathrm{ng} / \mathrm{L}$ ) instead of all 10 biomarkers when the Cox model was used (see online supplementary tables $3 \mathrm{~S}$ and $4 \mathrm{~S}$ ).

Table 3 summarises the ROC analyses. For prediction of the composite outcome (yes/no), the area under the ROC increases from 0.711 to 0.732 when the 10 novel biomarkers are added to the 'standard predictors', but almost all the marker information is contained in $\log$ (hs$\mathrm{cTnT} / \mathrm{ng} / \mathrm{L})$ and $\log (\mathrm{pro}-\mathrm{BNP} / \mathrm{ng} / \mathrm{L}) \quad(\mathrm{AUC}=0.730)$. The 'dynamic' $C$-index values are smaller as prediction of event times is more difficult, but the gains are similar. All-cause death shows the same general pattern.

\section{DISCUSSION}

In this study, we assessed the combined value of 12 newer biomarkers not routinely used in clinical work to predict all-cause death and a composite outcome (AMI, UAP, CeVD or all-cause death). We used a cut value of predicted risk $=0.5$ to separate correct predictions of the observed patient status from incorrect ones. When we combined the biomarkers with the 'standard predictors' routinely available for a general practitioner when he/she meets a patient with stable coronary artery disease (CAD), $84.7 \%$ of the survival status were correctly predicted. In case of the composite outcome the number was $68.4 \%$. In both cases, the combined contribution of the newer biomarkers amounted to $<1.5 \%$.

Our patients resemble those of The Prospective Observational Longitudinal Registry of Patients with Stable Coronary Heart Disease (CLARIFY) study, ${ }^{32}$ which enrolled 20291 patients. The CLARIFY patients had been observed with a median of 24.1 months. However, enrolment took place 10 years later than in the CLARICOR trial and the incidence of $\mathrm{CV}$ deaths or myocardial infarctions in these patients was considerably lower, ${ }^{32}$ probably reflecting improved quality of treatment and more frequent statin treatment in the CLARIFY patients $(84 \%$ compared with only $41 \%$ in the CLARICOR material). So, the age of our material is a weakness.

In our present study, we are using our data to develop a prediction model. Then we evaluate the performance using the same data that we used to develop the model. Clearly, this is bound to produce overly optimistic results compared with testing our model using independent data. But we argue that the aim of this study was not to present a prediction model but to assess the newer biomarkers' contribution to model performance when added on top of routinely available clinical and laboratory data. Therefore, if tested on independent data, the contribution of the newer biomarkers to prognosis of patients with stable $\mathrm{CAD}$ are likely going to be worse than observed here. 
Table 2 The two outcomes (1) all-cause death and (2) the composite outcome of AMI, UAP, CeVD or all-cause death were studied

\begin{tabular}{|c|c|c|c|}
\hline \multirow[b]{2}{*}{ Model and covariates included in model } & \multirow{2}{*}{$\begin{array}{l}\text { Total number of } \\
\text { predictions made } \\
\text { per outcome }\end{array}$} & \multicolumn{2}{|c|}{ Number and per cent of correct predictions of events } \\
\hline & & $\begin{array}{l}\text { All-cause death } \\
\text { N (\%) }\end{array}$ & $\begin{array}{l}\text { Composite of AMI, UAP, } \\
\text { CeVD or all-cause death N (\%) }\end{array}$ \\
\hline $\begin{array}{l}\text { Model 2: Cox model with } \\
\text { 'Standard predictors (SP)' added to model }\end{array}$ & 5972 & 4977 (83.3) & $4084(68.4)$ \\
\hline $\begin{array}{l}\text { Model 3: Cox model with } \\
\text { SP+10 newer biomarkers added to model }\end{array}$ & 5972 & $5056(84.7)$ & $4165(69.9)$ \\
\hline
\end{tabular}

AMI, acute myocardial infarction; CeVD, cerebrovascular disease; UAP, unstable angina pectoris.

\section{Methodology}

Regarding our methodology, the performance statistics reported here are minimal, but they suffice to show that the results are meagre. Prediction at 3, 6 and 9 years covers the follow-up as well as a sophisticated integral over continuous time.

\section{Strengths}

The strengths of the CLARICOR trial are the size of the patient population, the long duration of follow-up, few

\begin{tabular}{|c|c|c|}
\hline & $\begin{array}{l}\text { Binary } \\
\text { outcome C } \\
\text { (AUC) }\end{array}$ & Dynamic C \\
\hline & $\begin{array}{l}\text { Observed } \\
\text { (predicted)* }\end{array}$ & Observed $†$ \\
\hline \multicolumn{3}{|l|}{ Composite outcomeł (1115 events) } \\
\hline SP only & $0.711(0.707)$ & 0.640 \\
\hline The 10 newer markers and SP & $0.732(0.732)$ & 0.657 \\
\hline $\begin{array}{l}\log (\mathrm{hs}-\mathrm{cTnT} / \mathrm{ng} / \mathrm{L})+\log \text { (pro- } \\
\mathrm{BNP} / \mathrm{ng} / \mathrm{L})+\mathrm{SP}\end{array}$ & $0.730(0.730)$ & 0.656 \\
\hline \multicolumn{3}{|l|}{ All-cause death (644 deaths) } \\
\hline SP only & $0.792(0.793)$ & 0.737 \\
\hline The 10 newer markers and SP & $0.824(0.816)$ & 0.765 \\
\hline $\begin{array}{l}\text { log(hs-cTnT/ng/L)+log(pro- } \\
\text { BNP/ng/L)+SP }\end{array}$ & $0.821(0.813)$ & 0.762 \\
\hline
\end{tabular}

Cox model estimates applied to the 0-9years follow-up window $(n=1998)$.

‘The 'observed' AUCs summarise a ROC plot of cumulative events against cumulative non-events, with cumulation from large to small estimated risks. The corresponding 'predicted' AUC cumulates the predicted risks instead. Discrepancies between the two curves would suggest a model failure (calibration problems). The curves (not shown) were practically identical.

$\dagger$ Analogous concordance rate between time to event and predicted risk.

$\ddagger$ Composite outcome: first occurrence of acute myocardial infarction, unstable angina pectoris, cerebrovascular disease or death (table 1.

AUC, area under the curve; hs-cTnT, high-sensitive assay cardiac troponin T; ROC, receiver operating characteristics; SP, standard predictors. losses to follow-up (1\%), the ethnic homogeneity of the patient population (most being Caucasians), rarity of missing values, with focus on an operationally defined, homogeneous and relevant patient category. The design implies that the patients are sampled at random, presumably uneventful, time points during their stable state (as defined by the CLARICOR trial).

\section{Limitations}

Among those 7586 patients who declined our invitation to visit a cardiology centre, many must have been eligible for the CLARICOR trial, and we do not know how they looked and fared. With a response rate about $50 \%$, the cohort could represent a prognostic elite if responders were mostly mobile and health-conscious patients. So, selection bias cannot be excluded.

Furthermore, users of these data should remain aware of one feature: patients if any who became eligible for the CLARICOR trial during the period 1993 to 1999 and then died before August 1999 are absent. Thus, our data do not represent patients as they enter a stable disease state (as delimited by CLARICOR exclusion criteria); instead, they may be regarded as community patients (subject to some self-selection) seen by their physician or at an outpatient clinic on a random date during their stable state.

The patients recruited for the CLARICOR trial were diagnosed with CAD about 20 years ago. Because of the developments in treatment and rehabilitation, there has been a very significant and gradual improvement in the prognosis of such patients as shown in national data. ${ }^{33}$ Given these uncertainties, prognostic findings in the CLARICOR cohort may not be directly applied to present-day patients. However, the overall, somewhat disappointing, picture presented by the predictive performance of standard ${ }^{1}$ and newer biochemical predictors studied 10-20 years ago would hardly be much different if studied today.

Potential weaknesses of the present cohort within the context of prognostication of patients with stable CAD as here defined include the fact that only questionnaire data were collected at randomisation. No data are available concerning left ventricle function, body mass index, blood pressure and general health. These shortcomings are mitigated by the fact that, by design, the present study 
sees the patient in a situation where (s)he visits a physician for reasons unrelated to the coronary disease, as already stressed. In such situations, counselling and decisions must typically be made without access to echocardiography or other special investigations. Furthermore, if this information had been available, the prognostic gain we study would probably have been still poorer. Moreover, we included age, sex, hypertension, prior myocardial infarction, information about current medication which has previously been shown to be a fair replacement for prognostication instead of left ventricular ejection fraction. $^{34}$

It is noted that the patients studied by us were all in a stable state of their disease, without cardiac complaints. Therefore, one should not conclude from this study that the biomarkers studied here may not be useful in many other clinical contexts, although biomarkers have been shown to of modest help in evaluating $\mathrm{CV}$ risk assessment in asymptomatic people not suffering from CAD. ${ }^{35}$

\section{CONCLUSIONS}

In the present clinical context, the contribution of the 12 biomarkers not yet used in clinical routine work proved to be minimal. Furthermore, of the 10 statistically promising novel biomarkers all could be replaced by hs-cTnT and pro-BNP, possibly supplemented by OPG.

\section{Author affiliations}

${ }^{1}$ The Copenhagen Trial Unit, Centre for Clinical Intervention Research, Rigshospitalet, Copenhagen University Hospital, Copenhagen, Denmark ${ }^{2}$ Department of Regional Health Research, The Faculty of Heath Sciences, University of Southern Denmark, Copenhagen, Denmark

${ }^{3}$ Section of Biostatistics, Department of Public Health Research, University of Copenhagen, Copenhagen, Denmark

${ }^{4}$ Department of Cardiology, Hvidovre Hospital, Copenhagen University Hospital, Copenhagen, Denmark

${ }^{5}$ Cardiology, Herlev and Gentofte Hospital, Herlev, Denmark

${ }^{6}$ Department of Cardiology, Bispebjerg Hospital, Copenhagen University Hospital, Copenhagen, Denmark

${ }^{7}$ Rigshopitalet, University of Copenhagen, Copenhagen, Denmark

${ }^{8}$ Department of Clinical Microbiology, Odense University Hospital, Copenhagen,

Denmark

${ }^{9}$ Department of Cardiology, Herlev Hospital, Copenhagen University Hospital, Herlev, Denmark

${ }^{10}$ The Medical Research Laboratory, Department of Clinical Medicine, Aarhus University, Aarhus, Denmark

${ }^{11}$ Department of Medical Sciences, Uppsala University, Uppsala, Sweden

${ }^{12}$ Family Medicine and Primary Care, Karolinska Universitetssjukhuset, Stockholm, Sweden

Acknowledgements The authors would like to thank the CLARICOR trial participants. The authors would like to thank the investigators and other staff involved during the first phases of the CLARICOR trial (for full list of names, please see references 3,13 and 15 ). The authors would like to thank the original funders of the CLARICOR trial (see references 3, 13 and 15). The Copenhagen Trial Unit, Centre for Clinical Intervention Research, is thanked for providing monetary support for part of the biochemical analyses for the PREMAC study as well as wages for Per Winkel, Janus C. Jacobsen and Christian Gluud.

Contributors PW, JH, JCJ and CG contributed substantially to the concept and design and drafted the manuscript, PW and JH contributed equally to this paper, and conducted the statistical analyses. AL and JÄ conducted the analysis of lipids and creatinine. PW, JCJ, JH, GBJ, EK, AS, JK, HJK, KKI, MB, AL, JÄ and CG revised the manuscript critically for important intellectual content, gave final approval of version to be published and agreed to be accountable for all aspects of the work in assuring that questions related to the accuracy or integrity of any part of the work are appropriately investigated and resolved. PW, JCJ, JH, GBJ, EK, AS, JK, HJK, KKI, $M B, A L, J A ̈$ and $C G$ contributed substantially to the interpretation of the data.

Funding This study was funded by the Copenhagen Trial Unit, Centre for Clinical Intervention Research; original funders of the CLARICOR trial and The Swedish Research Council, Swedish Heart-Lung foundation; Thuréus Foundation; Marianne and Marcus Wallenberg Foundation, Dalarna University and Uppsala University.

Competing interests None declared.

Patient consent for publication Not required.

Ethics approval Ethics approval and consent to participate was given by the regional ethics committee (KF 01-076/99 and journal no. H-12012125), the Danish Medicines Agency (2612-975) and the Danish Data Protection Agency (1999-1200-174).

Provenance and peer review Not commissioned; externally peer reviewed.

Data availability statement Data will be available in a public, open access repository. All pertinent anonymised data will be uploaded at ZENODO (http:// zenodo.org/) when the individual manuscripts have been published.

Open access This is an open access article distributed in accordance with the Creative Commons Attribution Non Commercial (CC BY-NC 4.0) license, which permits others to distribute, remix, adapt, build upon this work non-commercially, and license their derivative works on different terms, provided the original work is properly cited, appropriate credit is given, any changes made indicated, and the use is non-commercial. See: http://creativecommons.org/licenses/by-nc/4.0/.

\section{ORCID iDs}

Janus Christian Jakobsen http://orcid.org/0000-0002-3642-2120

Christian Gluud http://orcid.org/0000-0002-8861-0799

\section{REFERENCES}

1 Winkel P, Jakobsen JC, Hilden J, et al. Prognostic value of routinely available data in patients with stable coronary heart disease. A 10-year follow-up of patients sampled at random times during their disease course. Open Heart 2018;5:e000808.

2 Winkel P, Jakobsen JC, Hilden J, et al. Predictors for major cardiovascular outcomes in stable ischaemic heart disease (PREMAC): statistical analysis plan for data originating from the CLARICOR (clarithromycin for patients with stable coronary heart disease) trial. Diagn Progn Res 2017;1:10.

3 Jespersen CM, Als-Nielsen B, Damgaard M, et al. Randomised placebo controlled multicentre trial to assess short term clarithromycin for patients with stable coronary heart disease: CLARICOR trial. BMJ 2006;332:22-7.

4 Hansen S, Als-Nielsen B, Damgaard M, et al. Intervention with clarithromycin in patients with stable coronary heart disease. Heartdrug 2001;1:14-19.

5 Carlsson AC, Ruge T, Kjøller E, et al. 10-Year associations between tumor necrosis factor receptors 1 and 2 and cardiovascular events in patients with stable coronary heart disease: a CLARICOR (effect of clarithromycin on mortality and morbidity in patients with ischemic heart disease) trial substudy. J Am Heart Assoc 2018;7:e 008299.

6 Wuopio J, Hilden J, Bring C, et al. Cathepsin B and S as markers for cardiovascular risk and all-cause mortality in patients with stable coronary heart disease during 10 years: a CLARICOR trial sub-study. Atherosclerosis 2018;278:97-102.

7 Harutyunyan MJ, Mathiasen AB, Winkel P, et al. High-Sensitivity $\mathrm{C}$-reactive protein and $\mathrm{N}$-terminal pro-B-type natriuretic peptide in patients with stable coronary artery disease: a prognostic study within the CLARICOR trial. Scand J Clin Lab Invest 2011;71:52-62.

8 Schroder J, Jakobsen JC, Winkel P, et al. Prognosis and reclassification by $\mathrm{YKL}-40$ in stable coronary artery disease. $J \mathrm{Am}$ Heart Assoc 2020;9:e014634.

9 Lyngbæk S, Winkel P, Gøtze JP, et al. Risk stratification in stable coronary artery disease is possible at cardiac troponin levels below conventional detection and is improved by use of N-terminal pro-Btype natriuretic peptide. Eur J Prev Cardiol 2014;21:1275-84.

10 Iversen KK, Teisner B, Winkel P, et al. Pregnancy associated plasma protein-A as a marker for myocardial infarction and death in patients with stable coronary artery disease: a prognostic study within the CLARICOR trial. Atherosclerosis 2011;214:203-8.

11 Larsen SB, Grove EL, Pareek M, et al. Calprotectin and platelet aggregation in patients with stable coronary artery disease. PLoS One 2015;10:e0125992. 
12 Bjerre M, Hilden J, Kastrup J, et al. Osteoprotegerin independently predicts mortality in patients with stable coronary artery disease: the CLARICOR trial. Scand J Clin Lab Invest 2014;74:657-64.

13 Winkel P, Hilden J, Hansen JF, et al. Clarithromycin for stable coronary heart disease increases all-cause and cardiovascular mortality and cerebrovascular morbidity over 10years in the CLARICOR randomised, blinded clinical trial. Int J Cardiol 2015;182:459-65.

14 Winkel P, Hilden J, Fischer Hansen J, et al. Excess sudden cardiac deaths after short-term clarithromycin administration in the CLARICOR trial: why is this so, and why are statins protective? Cardiology 2011;118:63-7.

15 Gluud C, Als-Nielsen B, Damgaard M, et al. Clarithromycin for 2 weeks for stable coronary heart disease: 6-year follow-up of the CLARICOR randomized trial and updated meta-analysis of antibiotics for coronary heart disease. Cardiology 2008;111:280-7.

16 Helmersson-Karlqvist J, Ridefelt P, Lind L, et al. Reference values for 34 frequently used laboratory tests in 80-year-old men and women. Maturitas 2016;92:97-101.

17 Inker LA, Schmid $\mathrm{CH}$, Tighiouart $\mathrm{H}$, et al. Estimating glomerular filtration rate from serum creatinine and cystatin C. $N$ Engl J Med 2012;367:20-9.

18 Wang N, Bai $X$, Jin B, et al. The association of serum cathepsin $B$ concentration with age-related cardiovascular-renal subclinical state in a healthy Chinese population. Arch Gerontol Geriatr 2016;65:146-55

19 Ärnlöv J, Ruge T, Ingelsson E, et al. Serum endostatin and risk of mortality in the elderly: findings from 2 community-based cohorts. Arterioscler Thromb Vasc Biol 2013;33:2689-95.

20 Jobs E, Ingelsson E, Risérus U, et al. Association between serum cathepsin S and mortality in older adults. JAMA 2011;306:1113-21.

21 Carlsson AC, Larsson TE, Helmersson-Karlqvist J, et al. Soluble TNF receptors and kidney dysfunction in the elderly. J Am Soc Nephrol 2014:25:1313-20.

22 Helmersson-Karlqvist J, Larsson A, Carlsson AC, et al. Urinary neutrophil gelatinase-associated lipocalin (NGAL) is associated with mortality in a community-based cohort of older Swedish men. Atherosclerosis 2013;227:408-13.
23 Kjøller E, Hilden J, Winkel P, et al. Good interobserver agreement was attainable on outcome adjudication in patients with stable coronary heart disease. J Clin Epidemiol 2012;65:444-53.

24 Kjøller E, Hilden J, Winkel P, et al. Agreement between public register and adjudication Committee outcome in a cardiovascular randomized clinical trial. Am Heart J 2014;168:197-204.

25 Helweg-Larsen K. The Danish register of causes of death. Scand J Public Health 2011;39:26-9.

26 Lynge E, Sandegaard JL, Rebolj M. The Danish national patient register. Scand J Public Health 2011;39:30-3.

27 Lin DY, Wei LJ, Ying Z. Checking the Cox model with cumulative sums of martingale-based residuals. Biometrika 1993;80:557-72.

28 Allison PD. Survival analysis using SAS: a practical guide. 2nd edn. NC, USA: SAS institute Inc, 2010.

29 Harrell FE, Lee KL, Mark DB. Multivariable prognostic models: issues in developing models, evaluating assumptions and adequacy, and measuring and reducing errors. Stat Med 1996;15:361-87.

30 Pencina MJ, D'Agostino RB. Overall C as a measure of discrimination in survival analysis: model specific population value and confidence interval estimation. Stat Med 2004;23:2109-23.

31 Little RJA. A test of missing completely at random for multivariate data with missing values. J Am Stat Assoc 1988;83:1198-202.

32 Steg PG, Greenlaw N, Tendera M, et al. Prevalence of anginal symptoms and myocardial ischemia and their effect on clinical outcomes in outpatients with stable coronary artery disease: data from the International observational clarify registry. JAMA Intern Med 2014;174:1651-9.

33 Schmidt M, Jacobsen JB, Lash TL, et al. 25 year trends in first time hospitalisation for acute myocardial infarction, subsequent short and long term mortality, and the prognostic impact of sex and comorbidity: a Danish nationwide cohort study. BMJ 2012;344:e356.

34 SolomonSD, ClaggettB, DesaiAS, et al Influence of ejection fraction on outcomes and efficacy of sacubitril/valsartan (Icz696) in heart failure with reduced ejection fraction. Circulation 2016;9:e00274.

35 Scott IA. Evaluating cardiovascular risk assessment for asymptomatic people. BMJ 2009;338:a2844. 